\title{
Hybrid Multi-Sensor Integration for Static or Dynamic Obstacle Detection, Tracking and Classification for Autonomous Vehicle
}

\author{
Dr. S. Rajkumar ${ }^{1 *}$, Aklilu Teklemariam²,Addisalem Mekonnen ${ }^{3}$ \&Habtamu \\ Tilahun $^{4}$ \\ ${ }^{1,2,3 \& 4}$ Department of Mechanical Engineering, Faculty of Manufacturing, \\ Institute of Technology, Hawassa University, Hawassa, Ethiopia. \\ E-Mail: ccetraj@gmail.com ${ }^{*}$,ake16tm@gmail.com ${ }^{2}$, \\ addisalemmekonnen9@gmail.com ${ }^{3}$ \&habteadom@gmail.com ${ }^{4}$
}

DOI: $10.51201 / J U S S T / 21 / 06424$

http://doi.org/10.51201/JUSST/21/06424

\begin{abstract}
Autonomous Vehicles (AV) reduces human intervention by perceiving the vehicle's location with respect to the environment. In this regard, utilization of multiple sensors corresponding to various features of environment perception yields not only detection but also enables tracking and classification of the object leading to high security and reliability. Therefore, we propose to deploy hybrid multi-sensors such as Radar, LiDAR and camera sensors. However, the data acquired with these hybrid sensors overlaps with the wide viewing angles of the individual sensors and hence convolutional neural network and Kalman Filter (KF) based data fusion framework was implemented in a goal to facilitate robust object detection system to avoid collisions in roads. The complete system tested over 1000 road scenarios for real-time environment perception showed that our hardware and software configurations outperformed numerous other conventional systems. Hence, this system could potentially find its application in object detection, tracking and classification in real-time environment.
\end{abstract}

Keywords: Autonomous Vehicle;Driver Assistance; Multi-sensor Environmental Perception;Data Fusion.

\section{Introduction}

Autonomous Vehicles (AV) are incorporated with technology that facilitates transportation accessibility and reduce cost which may benefit low-income countries for logistics and people with mobility issues $[1,2]$. AVs aim to reduce crash through interconnectivity of vehicles, meanwhile reducing pollution, congestion and energy consumption. The high cost of AVs and the involvement of multi-disciplinary research had hindered its commercialization even though it has been conceptualized a decade ago [3]. Hence, the overall expenditure for research and development amounts to 77 billion euros to nurture the innovation that disrupts automotive industry [4]. AVs become to reality after the inception of Google cars. Since then the technology has been a motive to the competitive car makers as listed in Table 1. Moreover, 50\% AV sales is forecasted for 2040 among all the vehicles [5]. However, it may cause unemployment due to the reduction in man power for transportation and logistics. Therefore, it is necessary to understand the challenges and embrace the opportunitiesin such technological disruption situations. 
One of the important features of AVs is to detect the objects on its vicinity being static or dynamic [6]. This can be achieved by incorporating sensors covering $360^{\circ}$ viewing angle with respect to the vehicle used in two categories. The first usage is to localize the vehicle with respect to the environment and the next is to perceive the environment using active sensors such as LiDAR, Radar, cameras. Once the localization and environment perception is made, the decision on the action is manipulated by the controllers and actuators as shown in Figure 1[7].Information from the different sensors needs integration and an accurate global representation of environment for obstacle detection. Hybrid Multi-sensor data fusion in AVs involves video, image and signal processing and considerable efforts have been provided in data fusion techniques $[8,9]$. These data fusion methodologies can be employed on the raw data, after feature extraction and on the decision levels that can be categorized: Low level fusion deals with fusing raw data from hybrid sensors to improve the detection accuracy; medium level fusion depends on fusing the features or characteristics of the object such as shape, dimension, orientation, velocity and position; high level fusion is also known as decision fusion in which certain decision about the obstacle by various sources are compared; multiple level fusion depends on the data fusion on any of the above fusion levels discussed [10-12]. LiDAR sensors and camera vision systems with different fusion algorithms have been studied. Some studies have performed single sensor and single fusion algorithms, multi hybrid sensors and single fusion [13]. Even though, there are numerous literature reported on the AV hardware and improvement in the accuracy obstacle detection by various fusion algorithms, the implementation feasibility of these algorithm has been less explored. Hence in our work, we have incorporated radar, LiDAR and camera to cover $360^{\circ}$ viewing angles and implemented fusion algorithms at medium-level. Meanwhile a trade-off between the system complexities and implementation in the real-world scenario was performed over 1000 situations and the environmental perception capability has been improved.

Table 1: Specification of AV by different car makers.

\begin{tabular}{|c|c|c|c|c|}
\hline BMW 5 Series & $\begin{array}{c}\text { Mercedes Benz S } \\
\mathbf{5 0 0}\end{array}$ & $\begin{array}{c}\text { Nissan Leaf } \\
\mathbf{E V}\end{array}$ & $\begin{array}{c}\text { Google Prius } \\
\text { and Lexus }\end{array}$ & $\begin{array}{c}\text { General Motors } \\
\text { Cadillac SRX }\end{array}$ \\
\hline $\begin{array}{c}\text { Radar Sensors } \\
\text { detect objects } \\
\text { ahead }\end{array}$ & $\begin{array}{c}\text { Stereo camera for } \\
\text { locating 3D } \\
\text { objects }\end{array}$ & $\begin{array}{c}\text { Front and } \\
\text { side radar }\end{array}$ & $\begin{array}{c}\text { LiDAR on the } \\
\text { roof of the car }\end{array}$ & Radar \\
\hline $\begin{array}{c}\text { Side laser } \\
\text { scanners }\end{array}$ & $\begin{array}{c}\text { Additional camera } \\
\text { for road signs }\end{array}$ & $\begin{array}{c}\text { Front, rear } \\
\text { and side laser } \\
\text { scanners }\end{array}$ & $\begin{array}{c}\text { Front and side } \\
\text { radar }\end{array}$ & Laser scanners \\
\hline $\begin{array}{c}\text { Differential GPS } \\
\text { Short and long } \\
\text { range radars }\end{array}$ & Camera & $\begin{array}{c}\text { measuring unit } \\
\text { for tracks } \\
\text { positioning }\end{array}$ & Cameras \\
\hline Ultrasonic & Ultrasonic sensors & $\begin{array}{c}\text { Four wide } \\
\text { angle } \\
\text { camensors }\end{array}$ & $\begin{array}{c}\text { Wheel encoder } \\
\text { tracks movement }\end{array}$ & Differential GPS \\
\hline
\end{tabular}




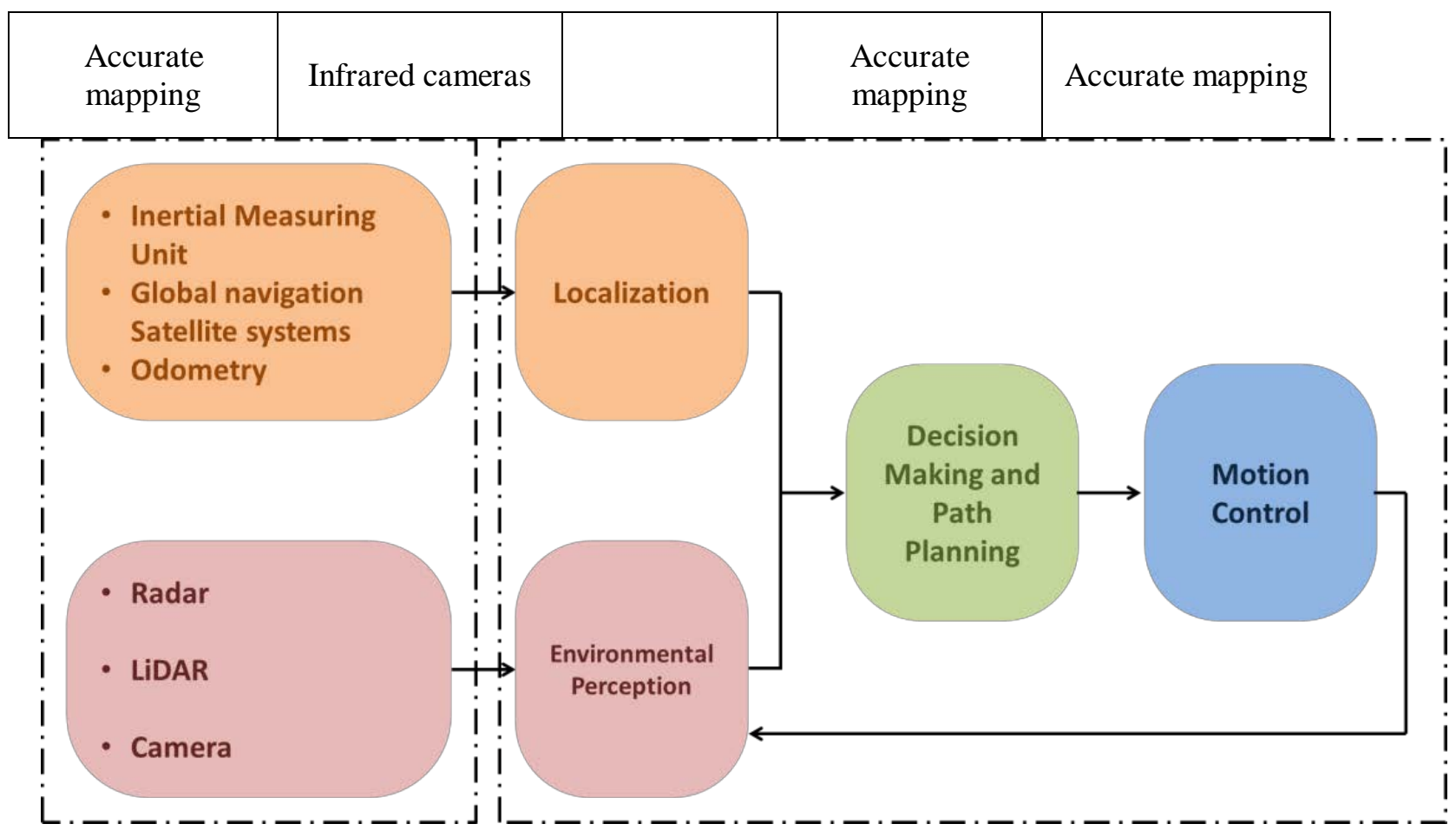

Figure 1: Architecture of Autonomous Vehicle. This work focuses on environmental perception.

\section{Materials and Methods}

For the identification of lane geometries on the road, several infrastructural changes including magnetic markers, aluminum-coated lane boundaries are necessary which is not suitable for worldwide implementation [14]. To eliminate the requirement of additional infrastructure for lane geometries and obstacle detection, the following approaches are suitable.

\subsection{Hardware}

Inertial Measuring Unit (IMU): The position and direction of the vehicle along with the road geometries can be identifies by inertial sensors and accurate mapping. Inertial sensors determine the continuous position and orientation [15].

Stereo Vision System (SVS): It includes radar, LiDAR and cameras for $360^{\circ}$ viewing angle. Radar has lower resolution thus resulting in the reduction of detection accuracy, the performance of LiDAR and camera is not upto the benchmark during rough weather and occlusion. Hence, the complementary sensors covering the complete surrounding acquire data with overlapping viewing angle where a data fusion would provide a reliable outcome of the obstacle as well as the lane geometry enabling smoother vehicle control [16].

\subsection{Fusion Algorithms}

The sensors installed may have their respective strength and weakness. Therefore, the data acquired once combined provides accurate information of the objects in the vicinity and lane geometry. The hybrid multi-sensor fusion algorithm consists of two parts as shown in Figure 2. The first section deals with obstacle classification, road segmentation and localization using LiDAR and cameras. Low level fusion is applied on the acquired data before road 
segmentation and obstacle distance estimation by convolutional neural networks (CNN). The second section detects objects and tracks their status using radar and LiDAR sensors. After road segmentation, i.e identifying the drivable area, the data acquired by the radar and LiDAR sensors are fused by Extended Kalman Filter (EKF) as the obstacles follow a non-linear motion [17]. Detection and tracking the obstacles prior on the road moderates the AV to take decision on path planning.

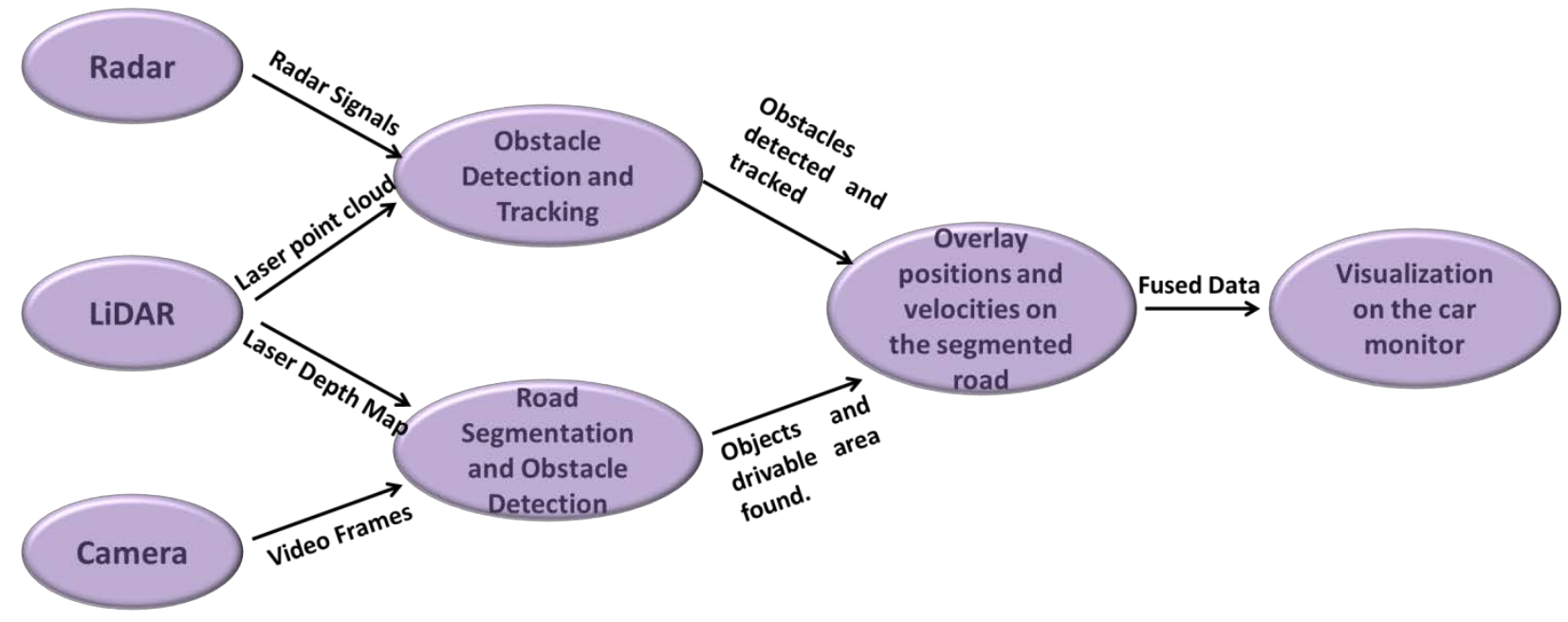

Figure 2: Hybrid Multi-Sensor Fusion Framework Pipeline.

\section{Results and Discussion}

Initially, the ground plane is defined by its normal vector and velocity with respect to the AV. Similarly the obstacles appearing above the ground plane are defined by its size, orientation, position and velocity as shown in Figure 3a. It provides not only the static but also the dynamic information of any obstacle. However, the performance of the camera may deteriorate under rough weather conditions during which the viewing angles of Radar and LiDAR supports the vision system. Hence the sensors should be capable of understanding its own limitations and communicates to the control system which is an important feature for the vision system to be reliable. However, the approaches followed in the literature identify 2D intensity patterns or features from a set of image sequence. The selection was usually made at identifying the patterns and features that are typical to the obstacles including vehicle shadows, their bounding edges and dark wheels [15].

Figure $3 \mathrm{~b}$ illustrates the detection of lower edge of vehicle shadow by the fusion of cacmra and LiDAR viewing angles using extended Kalman Filter. The region of interest determined is usually triangle and its width decreases with the increase in distance from the vehicle to the obstacle. Edge operator is performed on the region of interest and the edges are detected by applying appropriate threshold between light and dark edges. Further, by applying temporal filtering and additional features, the system exhibited significant detection and tracking of the obstacle. However, during certain unfavorable conditions including night time, sun dawn and rise, no shadow will fall below the vehicle leading to the misinterpretation of horizontal edges. Moreover, the obstacle identification falls below the certain category of predefined sets of typical obstacles on the road. Hence these results are not highly reliable and thus can be utilized as potential add-on feature obstacle detection. Hence, we intend to estimate the distance as well to avoid the obstacles by providing signals to the actuators such as pedals and steering wheels. 
(a)

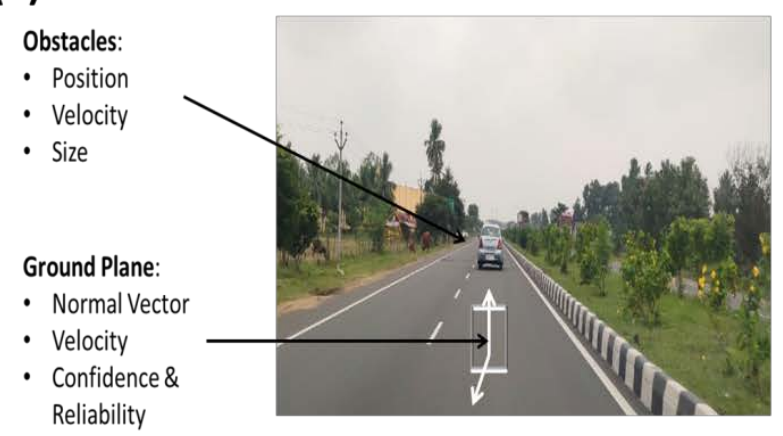

(b)

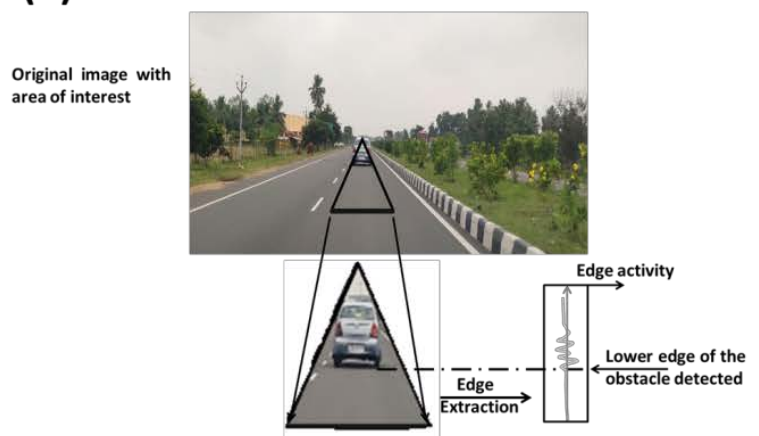

Figure 3: (a)Obstacle detection by defining the normal vector of the AV with respect to the ground plane and (b) Edge detection process of the obstacle by the fusion of camera and

\section{LiDAR data.}

The distance of the obstacle is identified using CNN. The initial part of the algorithm determines the region of interest as discussed earlier and draws a bounding box around the obstacle. When the obstacle is determined on the same vertical axis as the AV, the distance between them is estimated by calculating the number of pixels as shown in Table 2 and identification depends on predefined set of obstacles.

Table 2: Comparison of actual obstacle distance with respect to the distance estimated by our system.

\begin{tabular}{|c|c|c|}
\hline Sl. No & Actual Distance (cm) & $\begin{array}{c}\text { Predicted Distance using our } \\
\text { System (cm) }\end{array}$ \\
\hline 1 & 50 & 48 \\
\hline 2 & 75 & 71 \\
\hline 3 & 100 & 93 \\
\hline 4 & 125 & 119 \\
\hline 5 & 150 & 142 \\
\hline 6 & 175 & 168 \\
\hline
\end{tabular}

\section{Conclusion}

This work explored the static or dynamic obstacle detection in the line of path of an autonomous vehicle through the incorporation of radar, LiDAR and camera sensors. To gain accurate detection, multi-hybrid sensors were deployed in the AV vehicle and their overlapping viewing angles were fused by extended kalman filter at low level to seamlessly obtain detailed information of the scene on the road. The algorithm performs edge detection in the real time actual fused sensor data. After 1000 tests on the real road scenario, our system was found to be capable of detecting and identifying the obstacles with the predefined typical set, estimate the distance between the AV and obstacle which facilitates tracking the dynamics 
of the obstacle. This localization and symmetric environmental perception assists optimal path planning by providing optimal control input to the actuators of the AV. These results highlighted the improvement and performance gain of our new tracking system. However, further investigation on the contextual information of urban traffic environment perception, pedestrians walk etc. for improving the capability of our tracking system is required.

\section{References}

[1] Bagloee SA, Tavana M, Asadi M, Oliver T (2016) Autonomous vehicles: challenges, opportunities, and future implications for transportation policies. Journal of Modern Transportation 24: 284-303.

[2] Hong D, Kimmel S, Boehling R, Camoriano N, Cardwell W, Jannaman G, Purcell A, Ross D, Russel E (2008) Development of a semi-autonomous vehicle operable by the visuallyimpaired. In: IEEE International Conference on multisensor fusion and integration for intelligent systems, 2008. MFI 2008, pp. 539-544

[3] Guizzo E (2011) Howgoogle’s self-driving car works. IEEE Spectrum Online, October 18.

[4] Alia C, Gilles T, Reine T, Ali C (2015) Local trajectory planning and tracking of autonomous vehicles, using clothoid tentacles method. IEEE Intelligent Vehicles Symposium (IV).

[5] Jahromi BS, Tulabandhula T, Cetin S (2019) Real-Time Hybrid Multi-Sensor Fusion Framework for Perception in Autonomous Vehicles. MDPI Sensors 19:4357.

[6] Hossein TNN, Mita S, Long H (2010) Multi-sensor data fusion for autonomous vehicle navigation through adaptive particle filter. IEEE Intelligent Vehicles Symposium.

[7] Dima C, Vandapel N, and Hebert M (2003) Sensor and classifier fusion for outdoor obstacle detection: an application of data fusion to autonomous off-road navigation. Applied Imagery Recognition Workshop (AIPR2003). IEEE Computer Society, 255 - 262.

[8] Castanedo F (2013) A review of data fusion techniques,” The Scientific World Journal, vol. 2013.

[9] Luo R, Yih CC, and Su KL (2002) Multisensor fusion and integration: approaches, applications, and future research directions. Sensors Journal, IEEE, 2:107-119.

[10] Rubaiyat, AHM, Yaser F, Xin L, Gaurav B, Toyota I (2018) Multi-sensor Data Fusion for Vehicle Detection in Autonomous Vehicle Applications. Electronic Imaging, Autonomous Vehicles and Machines, 6:251-257.

[11] Xiao L, Dai B, Liu D, Hu T, Wu T. (2015) Crf based road detection with multi-sensor fusion. IEEE Intelligent Vehicles Symposium (IV) 192-198.

[12] Fagnant DJ, Kockelman K (2015) Preparing a nation for autonomous vehicles: opportunities, barriers and policy recommendations. Transp Res Part A 77:167-181

[13] Stiller C, Hipp J, Rossig C, Ewald A (2000) Multisensor obstacle detection and tracking. Image and Vision Computing 18: 389-396.

[14] Cho H, Seo YW, Kumar BVKV, Rajkumar R (2014) A Multi-Sensor Fusion System for Moving Object Detection and Tracking in Urban Driving Environments. IEEE International Conference on Robotics \& Automation (ICRA).

[15] Bedoya GO, Ferreria JV (2018) Sensor Fusion Tests for an Autonomous Vehicle, using Extended Kalman Filter. Journal of Engineering Science and Technology Review 11: 1-8. 